\title{
Vulvar schistosomiasis mansoni
}

\author{
Esquistossomose mansônica da vulva
}

José Roberto Lambertucci ${ }^{1}$, Izabela Voieta ${ }^{1}$ and Marina De Brot ${ }^{2}$

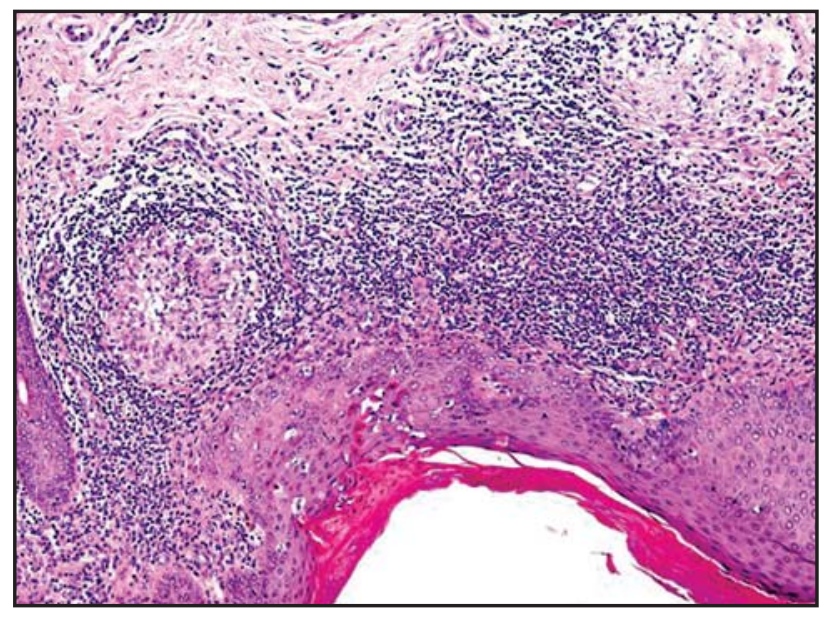

A

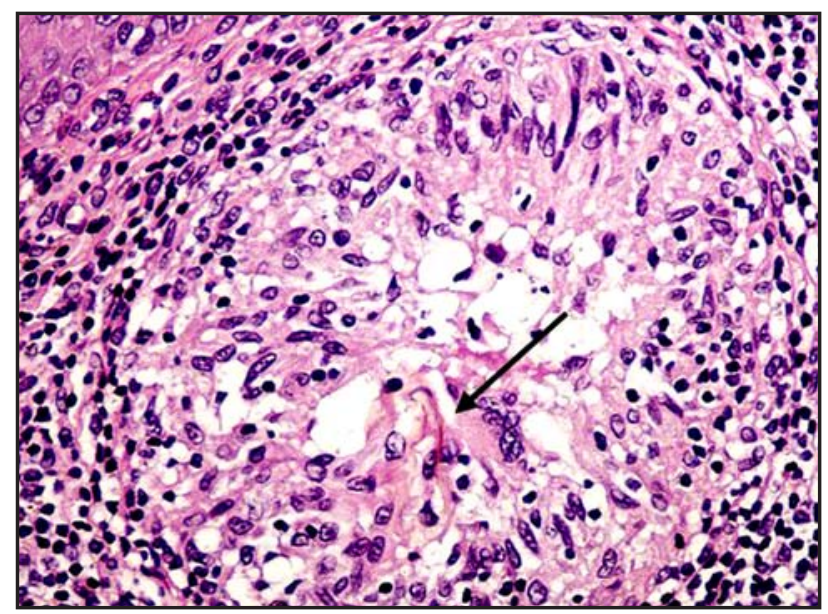

B

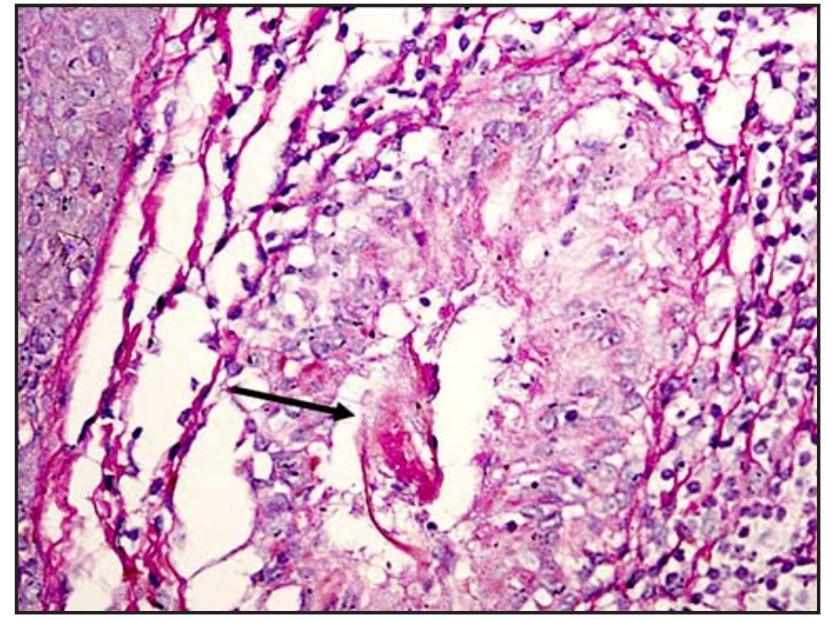

C

1. Curso de Pós-Graduação em Ciências da Saúde: Infectologia e Medicina Tropical, Faculdade de Medicina, Universidade Federal de Minas Gerais, Belo Horizonte, MG. 2. Serviço de Anatomia Patológica, Faculdade de Medicina, Universidade Federal de Minas Gerais, Belo Horizonte, MG.

Address to: Prof. José Roberto Lambertucci. Faculdade de Medicina/UFMG. Avenida Alfredo Balena 190, 30130-100 Belo Horizonte, MG, Brasil. e-mail: lamber@uai.com.br

Recebido para publicação em 12/09/2008

Aceito em 15/09/2008 
A 31-year-old female patient came to hospital complaining of an itchy nodular lesion $\left(1.6 \mathrm{~cm}^{3}\right)$ of the vulva (superior part of the right labium major) which appeared 8 years before. Occasionally, the lesion turned into an ulcer with minor bleeding, but the ulceration disappeared after topical treatment with steroids. During this period, she visited her gynecologist 4 times and was assured by him that the vulvar nodule was benign. In the last visit to her physician, the nodule was surgically excised and microscopic examination revealed the presence of a few well formed granulomata around Schistosoma mansoni egg shells (Figure A). Remnants of egg shells are pointed out in Figures B and C, under higher magnification (arrows). Intra-vaginal ultrasound did not find alterations in the uterus or ovaries. Abdominal ultrasound failed to demonstrate liver periportal thickening, characteristic of hepatosplenic schistosomiasis and 3 stool examinations remained negative for Schistosoma mansoni eggs. Notwithstanding, she was treat for schistosomiasis with praziquantel $(60 \mathrm{mg} / \mathrm{kg}$, body weight, oral single dose). Gynecological lesions caused by schistosomiasis continue to be underestimated in Brazil.

A paciente, de 31 anos, veio ao hospital porque apresentava lesão nodular $\left(1,6 \mathrm{~cm}^{3}\right)$ e pruriginosa na vulva (parte superior direita dos grandes lábios) que surgiu havia oito anos. Ocasionalmente, o nódulo tornava-se ulcerado e apresentava discreto sangramento, mas a ulceração desaparecia rapidamente após o uso de esteróides tópicos. Neste período, ela consultou o mesmo ginecologista por quatro vezes que lhe assegurou tratar-se de lesão de caráter benigno. Na última visita ao médico, o nódulo foi retirado cirurgicamente e ao exame microscópico evidenciou-se a presença de granulomas bem formados em torno de cascas de ovos de Schistosoma mansoni (Figura A). Restos de cascas de ovos estão assinalados nas Figuras B e C, em maior aumento (setas). 0 ultra-som endovaginal não revelou alterações em o útero ou nos ovários. 0 ultra-som do abdômen não revelou no fígado a presença de espessamento periportal característico da esquistossomose hepatoesplênica e três exames parasitológicos de fezes resultaram negativos para ovos de Schistosoma mansoni. Ainda assim, ela foi tratada para esquistossomose com praziquantel $(60 \mathrm{mg} / \mathrm{kg}$ de peso, em dose única oral). Lesões ginecológicas causadas pelo Schistosoma mansoni continuam sendo subestimadas no Brasil.

\section{REFERENCES}

1. Feldmeier H, Daccal RC, Martins MJ, Soares V, Martins R. Genital manifestations of schistosomiasis mansoni in women: important but neglected. Memórias do Instituto Oswaldo Cruz 93 (supl 1): 127-133, 1998.

2. Lambertucci JR, Serufo, JC, Gerspacher-Lara R, Rayes AA, Teixeira R, Nobre V, Antunes CM. Schistosoma mansoni: assessment of morbidity before and after control. Acta Tropica 77: 101-109, 2000.

3. Pratistadevi K, Ramdial AS. Transepithelial elimination of late cutaneous vulvar schistosomiasis. International Journal of Gynecological Pathology 20: 166-172, 2001. 